Bericht

\title{
BDDH-Symposium in Basel 2017: Zusammenfassung und Empfehlungen
}

\section{Relevanz der Thrombophiliediag- nostik für die Einschätzung des Thromboserezidivrisikos und mögliche therapeutische Konsequenzen}

Die tiefe Venenthrombose und Lungenarterienembolie sind häufig Folge kombinierter Einflüsse, sogenannter dispositioneller und expositioneller Risikofaktoren. Im Folgenden wird die Relevanz thrombophiler Risikodeterminanten für das Rezidivthromboserisiko anhand konkreter Risikoabschätzungen (in Absolutzahlen pro Jahr) dargelegt. Der Einfluss thrombophiler Risikofaktoren auf das Erstthromboserisiko unterscheidet sich relevant vom Einfluss auf das Rezidivthromboserisiko. Bezüglich Risikoabschätzungen zum Erstthromboserisiko wird auf zuvor publizierte Ausführungen verwiesen (1).

\section{Ermittlung des Thromboserezidiv- risikos nach Beendigung der antikoagulatorischen Therapie}

Die Thromboserezidivraten sind in den ersten 1-2 Jahren höher als im weiteren langfristigen Verlauf. Die im Folgenden angegebenen Rezidivraten beziehen sich auf das langfristige Risiko nach dem 2. Jahr nach Erstthrombose, da dieser Zeitabschnitt relevant für die Nutzen-Risiko-Abwägung im Vergleich zum langfristigen Risiko für schwere Blutungen unter Antikoagulation ist.

Die Art der Erstthrombose (idiopathische vs. reversible Risikokonstellation) hat eine wesentliche Bedeutung für das individuelle Rezidivrisiko. Patienten mit idiopathischer proximaler Erstthrombose oder Lungenembolie haben ein langfristiges jährliches Rezidivrisiko von ca. $3 \%$ (bei insgesamt $50 \%$ über 10 Jahre: ca. 10\%/Jahr in den ersten 2 Jahren, danach ca. $30 \%$ über 8 Jahre) $(2,3)$. Hingegen weisen Patienten mit einer Erstthrombose unter einer reversiblen Risikoexposition wie oraler Kontrazeption oder Operation ein jährliches Rezidivrisiko für eine Spontanthrombose von ca. 0-2\% auf. Je schwerwiegender der zur Thrombose füh- rende expositionelle Auslöser, umso geringer ist das spontane Thromboserezidivrisiko. Beispielsweise zeigt sich bei Patienten mit Erstereignis unter einer großen Operation ein Rezidivrisiko von 0-1\%/Jahr (2-4) und bei Patienten mit mildem temporärem Risikofaktor wie hormoneller Kontrazeption und ggf. auch Langstreckenflug ein jährliches spontanes Rezidivrisiko von ca. 1-2\% $(2,5-8)$. Im Vergleich zum Basisrisiko eines 60-jährigen Individuums von 1:1000/Jahr ohne stattgehabte Venenthrombose ist das absolute langfristige Thromboserezidivrisiko damit deutlich höher (ca. 30-fach höher bei spontaner Erstthrombose, ca. 10-20-fach höher bei Thrombose unter temporärem Risikofaktor), was eine hereditäre Komponente (z. B. heute noch unbekannte thrombophile Risikofaktoren) als zusätzliche Ursache wahrscheinlich macht, auch wenn sich diagnostisch kein Hinweis auf bekannte thrombophile Risikodeterminanten ergibt.

Thrombophile Risikofaktoren beeinflussen das Thromboserezidivrisiko ( $\triangleright$ Tab. 1). Um bisher nicht identifizierbare unbekannte Risikofaktoren bei der Risikostratifizierung für zukünftige Ereignisse zu berücksichtigen, muss anhand der Art der Erstthrombose das statistisch mittlere Rezidivrisiko abgeschätzt werden (z. B. langfristig 3\%/Jahr bei idiopathischer Erstthrombose). Eine darauf aufbauende individuelle Risikoabschätzung (in Prozent pro Jahr) für ein Thromboserezidivereignis nach spontaner proximaler Erstthrombose oder Lungenembolie kann durch Hinzunahme patientenspezifischer relativer Risiken ermittelt werden (z. B. Geschlecht, D-Dimer-Erhöhung, hereditäre Risikofaktoren). Das relative Risiko wird mit dem Basisrezidivrisiko multipliziert. Wie in $>$ Tabelle 1 dargestellt, führt z. B. ein erhöhter D-DimerWert zu einem 2-2,5-fachen Rezidivrisiko mit einer Steigerung von 3\% (Basisrisiko) auf 6\%/Jahr. Auf der Grundlage relativer Risiken und der Kenntnis des Basisrezidivrisikos können individuelle Absolutrisiken für Rezidivthrombosen angegeben werden, die die Grundlage für eine individuelle Therapieentscheidung bezüglich einer langfristigen ora- len Antikoagulation darstellen. Die in $\mathbf{T a}$ belle 1 angegebenen Risikoabschätzungen gelten nur für Patienten mit spontaner proximaler Erstthrombose oder Lungenembolie und nicht für Erstthrombosen mit relevantem Auslöser (Operation, Trauma etc.).

Individuelle Nutzen-RisikoAbwägung einer langfristigen oralen Antikoagulation unter Berücksichtigung des

Blutungsrisikos und thrombophiler Risikodeterminanten (nach spontaner proximaler tiefer Venenthrombose oder Lungenembolie)

Eine individuelle Nutzen-Risiko-Abwägung erfolgt unter Ermittlung des patientenspezifischen Risikos für schwere Blutungen unter oraler Antikoagulation mit Vitamin-K-Antagonisten im Vergleich zum thromboembolischen Rezidivrisiko. Die jeweiligen Risiken werden im Folgenden vergleichend gegenübergestellt.

\section{Risiko einer schweren}

Blutungskomplikation unter oraler Antikoagulation

Die langfristige orale Antikoagulation mit Vitamin-K-Antagonisten ist mit einem relevanten Risiko schwerer bzw. letaler Blutungskomplikationen assoziiert, welches durch Alter und Komorbiditäten bzw. Begleitmedikation weiter ansteigt. Das jährliche Blutungsrisiko liegt bei ca. 3\% in durchschnittlichen älteren Patientenkollektiven mit 2 Risikofaktoren (davon z. B. Alter > 65 Jahre, abgelaufene Blutung, Hirninsult, Vorliegen einer oder mehrerer Begleiterkrankungen (Diabetes mellitus, Hypertonie, Niereninsuffizienz (Kreatinin > 1,5mg/dl), Myokardinfarkt, Anämie (HKT < 0,30\%)). In gut kontrollierten Kollektiven mit bis zu einem Blutungsrisikofaktor ist von einer jährlichen Rate an Blutungskomplikationen von ca. $1 \%$ und bei Patienten mit $>3$ Risikofaktoren von ca. $10 \%$ auszugehen. Die Letalität der schweren Blutungen liegt bei 10\%, somit liegt die jährliche Rate letaler Blutungskomplikationen unter oraler Antikoagulation beim durchschnittlichen älteren Patienten mit 2 Risikofaktoren bei 0,3\% (9-15). Bei der Entscheidung zur langfristigen oralen Antikoagulation ist das individuelle Blutungsrisiko 
des Patienten unter Nutzen-Risiko-Abwägung zu berücksichtigen. Neben der initialen Bewertung ist das Blutungsrisiko im weiteren Verlauf in regelmäßigen, z. B. jährlichen Abständen in Abhängigkeit vom individuellen Krankheitsverlauf, dem Neuauftreten von Risikofaktoren einer Blutung und der Qualität der Einstellung einer oralen Antikoagulation zu reevaluieren.

\section{Empfehlungen zur Dauer der} oralen Antikoagulation nach Leitlinien (ohne Berücksichtigung thrombophiler

\section{Risikodeterminanten)}

Die aktuellen Leitlinienempfehlungen (13, 16, 17) zur Dauer einer Antikoagulation nach tiefer Venenthrombose und/oder
Lungenarterienembolie beruhen maßgeblich auf der Art der Erstthrombose (mit Auslöser vs. idiopathisch) und der Lokalisation (distal vs. proximal). Weiterhin werden die Persistenz eines Risikofaktors (z. B. Malignom) und die Patientenpräferenz berücksichtigt. Alle Patienten mit proximaler tiefer Venenthrombose und/oder Lungenarterienembolie sollten mindestens eine

Tab. 1 Langfristiges Risiko für Rezidivthrombose nach spontaner tiefer Venenthrombose (TVT) oder Lungenembolie (LE) in Abhängigkeit von der Art des Erstereignisses und der thrombophilen Risikodeterminanten: Vorschlag zur individuellen langfristigen oralen Antikoagulation (OAK) mit Vitamin-K-Antagonisten*

\begin{tabular}{|c|c|c|c|c|c|}
\hline & & $\begin{array}{l}\text { Spontanes (idiopathi- } \\
\text { sches) thromboemboli- } \\
\text { sches Erstereignis }\end{array}$ & \multicolumn{3}{|c|}{$\begin{array}{l}\text { Indikation zur oralen Antikoagulation } \\
\text { (nach Blutungsrisiko) (OAK) }\end{array}$} \\
\hline & & Rezidivrisiko/Jahr & \multicolumn{3}{|c|}{ Blutungsrisiko (nach Blutungsrisikofaktoren) (RF) \# } \\
\hline Risikofaktor & Relatives Risiko ** & $\begin{array}{l}\text { Absolutes Risiko } \\
\text { Im Mittel ca. 45\% } \\
\sim 10 \% \text { (1. Jahr) } \\
\sim 5-10 \% \text { (im 2. Jahr) } \\
\sim 3 \% \text { (ab 3. Jahr) }\end{array}$ & $\begin{array}{l}\text { Niedrig-mittel } \\
1 \% \\
\text { (0-1 RF) }\end{array}$ & $\begin{array}{l}\text { Hoch } \\
3 \% \\
(2-3 \mathrm{RF})\end{array}$ & $\begin{array}{l}\text { Sehr hoch } \\
10 \% \\
(\geq 4 \text { RF) }\end{array}$ \\
\hline Distale TVT & 0,5 & $2 \%$ & Keine OAK & \multirow[t]{3}{*}{ Keine OAK } & \multirow[t]{6}{*}{ Keine OAK } \\
\hline $\begin{array}{l}\text { Basisrisiko } \\
\text { (ohne thrombophile Risikofaktoren) } \\
\text { (bei Frauen eher 2,5\% pro Jahr) \#\# }\end{array}$ & 1 & $3 \%$ & \multirow[t]{5}{*}{ OAK } & & \\
\hline $\begin{array}{l}\text { Faktor-V-Leiden- oder Prothrombin } \\
\text { G20210A-Mutation heterozygot } \\
\text { Männliches Geschlecht } \\
\text { Residuelle Thrombose proximal; Rezidiv- } \\
\text { thrombose }\end{array}$ & $\sim 1,3 \%-1,6 \%$ & $4 \%-5 \%$ & & & \\
\hline $\begin{array}{l}\text { - D-Dimere erhöht (> } 500 \mathrm{ng} / \mathrm{ml}) \| \\
\text { - Protein-C/S-Mangel } \\
\text { - Faktor-V-Leiden- und Prothrombin } \\
\text { G20210A heterozygot } \\
\text { - Faktor-V-Leiden-Mutation homozygot *** } \\
\text { - Antithrombin-Mangel } \\
\text { - Antiphospholipid-Syndrom }\end{array}$ & $\sim 2 \%-2,5 \%$ & $\sim 6 \%-8 \%$ & & \multirow[t]{3}{*}{ OAK } & \\
\hline Maligner Prozess & $\sim 3$ & $\sim 9 \%$ & & & \\
\hline Faktor VIII > 234 IU/dl & $\sim 6$ & $18 \%$ & & & \\
\hline
\end{tabular}

TVT = tiefe Venenthrombose * Weiterführende Literaturangaben siehe (3). Die angegebenen absoluten Rezidivraten pro Jahr sind abgeleitet aus dem relativen Risiko der thrombophilen Risikodeterminante und dem Basisrisiko (bestimmt aufgrund der Zeit nach Erstereignis (>1 Jahr) und Art des Erstereignisses (idiopathisch). Es handelt sich damit nur um Schätzwerte, die jeweils einer kritischen individuellen Bewertung bedürfen.

\# Risikofaktoren für Blutungen: Alter $>65$ Jahre, anamnestisch Hirninfarkt, Diabetes, Leber- oder Niereninsuffizienz, Myokardinfarkt, Anämie (HKT $<30 \%$ ), anamnestisch Blutung, Malignom, Thrombozytopenie, antithrombozytäre Medikation, Einnahme von Schmerzmitteln/NSAIDs, labile INR, Alkoholkonsum, kürzlich Operation, Sturzneigung.

| Neuere und große Studien wurden stärker bei der Festlegung des relativen Risikos für eine Rezidivthrombose gewichtet.

** Die relativen Risiken des Risikofaktors beziehen sich auf das Thromboserezidivrisiko und nicht auf das relative Risiko des Risikofaktors für Erstthrombosen, das deutlich höher liegt.

\#\# Indikation zur langfristigen oralen Antikoagulation mit Vitamin-K-Antagonisten bei Frauen fraglich, sofern D-Dimer-Werte langfristig im Normbereich. Bei Therapie mit NOAKs/DOAKs in reduzierter Dosierung eher Indikation zur langfristigen Antikoagulation.

|| D-Dimere reproduzierbar erhöht nach Beendigung der oralen Antikoagulation.

${ }^{* * *}$ Nach eigenen Daten. In einer Publikation von Lindmarker et al. (19) wurde ein relatives Risiko von 4,1 ermittelt. Nach Ansicht der Autoren dürfte das wahre relative Risiko eher unter 3 liegen und damit mit dem Risiko anderer relevanter thrombophiler Risikofaktoren (z.B. Antithrombin-Mangel) besser vereinbar sein. 
3-6-monatige Antikoagulation erhalten. Bei Vorliegen eines relevanten Auslösers (z. B. Operation, Trauma, Estrogeneinnahme, Schwangerschaft) oder bei isoliert distaler Venenthrombose ist keine längere Antikoagulation indiziert.

In den ACCP-Guidelines wird nur bei Patienten mit spontaner proximaler Erstthrombose oder Lungenarterienembolie aufgrund des langfristig höheren Thromboserezidivrisikos (50\% über 10 Jahre, ca. $3 \% / J a h r$ ab 3. Jahr) eine langfristige orale Antikoagulation empfohlen, sofern das Blutungsrisiko niedrig ist $(\leq 1 \% / J a h r)$. Ein niedriges Blutungsrisiko besteht, wenn bei dem Patienten nicht mehr als ein Blutungsrisikofaktor vorliegt ( Tab. 1). Liegt ein hohes Blutungsrisiko vor ( $\geq 2$ Blutungsrisikofaktoren), wird auf eine langfristige orale Antikoagulation unter Nutzen-Risiko-Abwägung verzichtet $(13,16)$.

Individuelle Nutzen-Risiko-Abwägung einer langfristigen oralen Antikoagulation unter Berücksichtigung thrombophiler Risikodeterminanten (nach spontaner proximaler tiefer Venenthrombose oder Lungenembolie)

Milde thrombophile Risikodeterminanten (z. B. heterozygote Faktor-V-Leiden- oder Prothrombin-G20210A-Mutation) beeinflussen das Thromboserezidivrisiko nach spontan aufgetretener tiefer Venenthrombose oder Lungenembolie nur geringgradig (ca. 1,3-1,5-fache Risikosteigerung) ( Tab. 1). Demgegenüber können schwerere thrombophile Risikofaktoren das Thromboserezidivrisiko verdoppeln oder verdreifachen, $d$. h. es steigt von jährlich $3 \%$ ohne thrombophilen Risikofaktor auf 6-8\% und mehr an. Schwere thrombophile Risikofaktoren mit derartiger Steigerung des Thromboserezidivrisikos sind Mangel an Antithrombin oder Protein C, kombinierte oder homozygote Defekte von Faktor-V-Leiden- und ProthrombinG20210A-Mutation, Antiphospholipidantikörper sowie persistierende D-Dimer-Erhöhung ( Tab. 1). Das Vorliegen schwerer thrombophiler Risikofaktoren kann deswegen eine langfristige orale Antikoagulation nach spontaner tiefer Venenthrombose/ Lungenembolie unter Nutzen-Risiko-Abwägung begründen (gilt nicht für Thrombosen unter relevantem Auslöser wie Operation).

Eine individuelle Nutzen-Risiko-Abwägung erfolgt unter Ermittlung des patientenspezifischen Blutungsrisikos unter oraler Antikoagulation im Vergleich zur Abschätzung des thromboembolischen Rezidivrisikos. In $>$ Tabelle 1 wird das absolute jährliche Rezidivrisiko für Spontanthrombosen dem Blutungsrisiko gegenübergestellt und eine Antikoagulation dann empfohlen, wenn das individuelle Thromboserisiko das Blutungsrisiko deutlich übersteigt. Beispielsweise wird man einen Patienten mit einer spontanen proximalen Thrombose oder Lungenembolie (jährliches Thromboserezidivrisiko 3\% nach dem 2. Jahr) langfristig oral antikoagulieren, wenn das jährliche Blutungsrisiko niedrig ist $(\leq 1 \%)$, jedoch nicht antikoagulieren, wenn das Blutungsrisiko hoch ist ( $\geq 3 \%$ ). Trägt der Patient allerdings einen relevanten thrombophilen Risikofaktor (z. B. Mangel an Antithrombin oder homozygote Faktor-V-Leiden-Mutation) mit einem jährlichen Thromboserezidivrisiko von $\geq$ $6 \%$, so kann eine langfristige orale Antikoagulation auch bei einem Blutungsrisiko von $3 \%$ sinnvoll sein.

Eine Indikation zur langfristigen oralen Antikoagulation kann für Patienten mit „getriggertem“ Erstereignis auch bei Vorliegen thrombophiler Risikofaktoren nicht sicher abgeleitet werden. Es ist fraglich, ob schwere thrombophile Risikofaktoren wie Antithrombin-Mangel, homozygote Faktor-V-Leiden-Mutation oder Antiphospholipid-Antikörper eine Indikation zur langfristigen oralen Antikoagulation in dieser Patientengruppe darstellen. Bei einem Thromboseereignis nach Operation mit einem jährlichen Thromboserezidivrisiko von ca. $0,5 \%$ oder weniger bedingt auch eine Verdreifachung des Rezidivrisikos im Vergleich zum Blutungsrisiko unter oraler Antikoagulation keinen Vorteil für eine langfristige Antikoagulation. Dies entspricht auch aktuellen britischen Leitlinien zum Antiphospholipidsyndrom sowie den gültigen ACCP-Guidelines $(13,16,18)$. Die Empfehlung der deutschen Leitlinien (17) zur regelhaften langfristigen oralen Antikoagulation bei Thrombosepatienten mit schweren thrombophilen Risikofaktoren unabhängig von der Art des Auslösers (d. h. auch bei postoperativen Thrombosen) ist aufgrund der niedrigen absoluten Thromboserezidivrisiken nach postoperativer Erstthrombose eher kritisch zu sehen. Bei Trägern schwerer thrombophiler Risikofaktoren und Thrombosen mit milden Auslösern (z.B. Flugreise) ist eine langfristige orale Antikoagulation wahrscheinlich vertretbar. Hier fehlen leider detaillierte Daten zum Thromboserezidivrisiko stratifiziert nach verschiedenen milden Auslösern.

\section{Risikostratifizierung bei Verwendung von NOAKs/DOAKs}

Die Empfehlungen in Tabelle 1 beziehen sich auf eine Therapie mit Vitamin-K-Antagonisten. Bei Einsatz direkter oraler Antikoagulanzien ist von einem niedrigeren Blutungsrisiko auszugehen, insbesondere bei langfristiger Anwendung mit reduzierten Dosierungen nach den ersten 6 Monaten nach Thrombose. Damit dürfte nach Ansicht des Autors bei proximalen Thrombosen oder Lungenembolien nicht nur bei spontanen Thromboembolien, sondern auch unter milden Auslösern wie hormonellen Kontrazeptiva (Thromboserezidivrisiko ca. $1 \% / J a h r)$ bei Vorliegen schwerer thrombophiler Risikofaktoren eine langfristige orale Antikoagulation sinnvoll sein (Rezidivrisikosteigerung von $1 \%$ auf ca. $2-2,5 \% / J a h r)$.

Priv.-Doz. Dr. med. R.B. Zotz, Institut für Laboratoriumsmedizin, Blutgerinnungsstörungen und Transfusionsmedizin, LBT Zotz/Klimas

Literatur beim Verfasser

Impressum
Verantwortlich für den Inhalt:
Dr. med. Ute Scholz, BDDH-Vorsitzende
Vorstand des Berufsverbands der Deutschen
Hämostaseologen (BDDH e.V.)
Struempellstraße 40, 04289 Leipzig

